\section{Regulatory genes in fungi}

from a Correspondent

THE paper by Arst in this issue of can be simultaneously subject to more Nature (page 231) marks another step than one system of integrative regulain our understanding of regulation of tion. Arst and Cove and their colleages gene action in the mould Aspergillus (for example, Molec. gen. Genet., 126, nidulans. In 1970, Hynes and Pateman 111-141; 1973) had already described (Molec. gen. Genet., 108, 97-106, 107- in Aspergillus a regulator gene, called 116) reported mutants which identified are $A$, which is concerned with nitrogen a gene whose product seemed to act as catabolite repression, and which regua positive regulator of the structural lates the action of several other genes gene for amidase (amdS). Now Arst with functions in the generation of reports that this regulatory gene- ammonium ion. A type of mutant now called int $A$ for integrator-also allele, called $\operatorname{are} A^{r}$, represses these regulates expression of two genes con- other genes (including the amidase cerned with $\omega$-amino acid metabolism. gene) even in the absence of ammonia. These latter two genes, gat $A$ and gabA, "Constitutive" alleles of int $A$ can nulcode, respectively, for an inducible lify the repressing effect of are $A^{r}$, the transaminase acting on $\beta$-alanine, $\gamma$ - positive integrative control in this case aminobutyrate or $\delta$-aminovalerate and over-riding the negative. In addition, a permease for the same $\omega$-amino acids. one can have positive gene-specific The three genes under control are all regulation, acting in parallel with the unlinked to int $A$ and to each other. It "integrator" regulation. Thus, another seems that various mutations in int $A$ regulatory gene, amd $A$, is found by can affect the control of amdS, gat $A$ Arst to act positively on amdS (more and gabA differentially, and can also or les $s$ additively with int $A$ ) with no be associated with changes in the rela- effect on gabA and gat $A$.

tive responses to $\gamma$-aminobutyrate and The regulation of nitrogen metabol$\beta$-alanine as effectors. The conclusion is ism in Aspergillus, thanks mainly to that the int $A$ product interacts with the work of J. A. Pateman in Glasgow, each of the three genes (or with con- Arst (with D. J. Cove and C. Scazzoctrolling regions associated with them) chio) in Cambridge, M. J. Hynes in and also with various small molecular Australia and P. Weglenski in Warsaw, effectors, and that these specificities can is becoming an extremely complex be altered by mutation. Arst draws story-excessively so, perhaps, in the attention to the similarity between opinion of some. But the complexity is int $A$ and the type of integrator gene there in the eukaryotic cell. Attempts proposed by Britten and Davidson in to unravel the network of gene inter1969 (Science, 165, 349-357) as part of actions are bound to produce somean influential general model for regu- thing of a tangle at the outset, but perlation of gene action in eukaryotes.

Arst's report, together with earlier work, shows that sets of unlinked genes

Grosjean et al. were able to monitor in temperature jump relaxation experiments. This absorbance change shows the right spectral distribution to be expected from the base pairs presumed to be formed, and is not manifest when the two anticodons are not complementary. Moreover, the three complementary bases must be centred in the middle of each seven-membered anticodon loop for the reaction to be appreciably manifest. Thus, for interaction, the anticodons per se must be complementary, and complementarity between other bits of the anticodon loops does not help.

Returning to the temperature jump results, the authors found that the rate constant for the association reaction was close to that expected for the association of complementary trinucleotides, and, furthermore, virtually temperature independent. The surprise comes in the rate constant for the back dissociation reaction which was six orders of magnitude slower than expected. It follows that the equilibrium constant is $10^{8}$ times higher than expected.

More detailed analysis showed that the entropy of interaction was not very different from that expected for trinucleotide interaction, but the enthalpy change was about -25 kcalories, which is about 10 kcalories larger than expected. This difference of $10 \mathrm{kcalo}-$ ries is sufficient to account for the factor at $10^{6}$ and is at the kernel of the enhanced anticodon-anticodon interaction. This in itself suggested that stacking interactions were involved.

Pushing the investigation further, Grosiean et al. studied the interaction of Escherichia coli $\mathrm{tRNA}^{\mathrm{in}}$ with yeast tRNA ${ }^{\text {Phe }}$ in the presence of EDTA, in which conditions the former species is in the denatured conformational state. The association was still observed, suggesting that the structural features which distinguish the two conformer states are not important to the association. Similarly the removal by specific cleavage of the first 16 nucleotides from the $5^{\prime}$ end of the tRNA ${ }^{\text {Phe }}$ did not alter the interaction. On the other hand, fragments obtained from both these species such that anticodon helical arms could not form, still associated but with a constant reduced by about two orders of magnitude-leaving an enhancement of four orders still to be accounted for.

The remaining factors were deemed to reside in the "dangling ends" or the residual four bases in the anticodon loop that are not involved in the anticodon itself. Grosjean et al. point out that the sixth base in the anticodon loop (that is the one at the $3^{\prime}$ end of the anticodon) is frequently modified. They go on to show that the association constant varies for species with similar anticodons but differs in the nature of the sixth base, and, in particular, that excision of the $Y$ base from this position in $\mathrm{tRNA}^{\text {Phe }}$ leads to a substantial reduction in the enthalpy of association.

Grosiean et al. tie all these observations together by postulating that the enhanced association constant and the increased enthalpy of association originates from the stacking of the two anticodon arm helices on either side of the helical region formed by the anticodon-anticodon interaction, with the $3^{\prime}$ dangling ends stacked up as jam in this three layer cake. I choose this analogy carefully since jam is well known to be sticky.

We are finally left with the thought that something similar happens in the codon-anticodon interaction. Maybe the ribosome holds the relevant portion of the messenger in a configuration similar to that in the tRNA and allows the interaction to be similarly stabilised by stacking.

These results also imply that the anticodion helical arm exists in both the native and denatured forms of $E$. coli tRNA ${ }^{\mathrm{Glu}}$. Further evidence of the similarities and differences between these two forms of tRNA comes from a paper by Jones, Kearns and Muench (J. molec. Biol., 103, 747: 1976) who worked with $E$. coli tRNA ${ }^{\text {Trp }}$. They measured the low field nuclear magnetic resonance (NMR) spectrum of the native form of this species and assigned to their satisfaction all parts of the $11.5-14.5$ p.p.m. region to one or other structural feature expected of the clover leaf model in its surmised tertiary structure. There were thus 19 base pains, a protected $U$ or $G$ and two tertiary base pairs including $A_{14}-s^{4} U_{8}$. The agreement between their experimental spectrum and that computed from these assignments was very process for the denatured form and considered in detail the difference NMR spectrum between the two. They reasonable. They then repeated the 